\title{
Signal Amplification in NbN Superconducting Resonators via Stochastic Resonance
}

\author{
Baleegh Abdo* Eran Arbel-Segev, Oleg Shtempluck, and Eyal Buks \\ Department of Electrical Engineering, Technion, Haifa 32000, Israel
}

(Dated: April 19, 2018)

\begin{abstract}
We exploit nonlinearity in $\mathrm{NbN}$ superconducting stripline resonators, which is originated by local thermal instability, for studying stochastic resonance. As the resonators are driven into instability, small amplitude modulated (AM) microwave signals are amplified with the aid of injected white noise. The dependence of the signal amplification on the modulation amplitude and the modulation frequency is examined and compared with theory.
\end{abstract}

PACS numbers: 74.40.+k, 02.50.Ey, 85.25.-j

The notion that certain amount of white noise can appreciably amplify small periodic modulating signals acting on bistable systems, generally known as stochastic resonance, has been over the last two decades of a great interest 1, 2, 3, 4, 5]. It has been applied for instance to account for the periodicity of ice ages occurring on earth by Benzi and collaborators 6, 7]. It was demonstrated experimentally in several electronic devices such as ac-driven Schmitt triggers [8]. It has a growing role in explaining some important neurophysiological processes in neuronal systems [9, 10]. In addition, it was used to amplify small signals in various nonlinear systems, e. g. the intensity of one laser mode in a bistable ring laser 11], the magnetic flux in a superconducting quantum interference device [12, 13], and even more recently, a small periodic drive of a nanomechanical oscillator 14]. In this work we exploit the metastability exhibited by our nonlinear $\mathrm{NbN}$ superconducting stripline resonators in order to amplify a small applied sinusoidal modulating signal using stochastic resonance. Moreover, we apply stochastic resonance measurements in order to examine the dependence of the signal amplification generated by the resonator system on both the modulation frequency and amplitude.

The layout of the center layer of the $\mathrm{NbN}$ stripline resonator that was used is shown at the top-right corner of Fig. 2 Fabrication details as well as nonlinear characterization and modeling of these resonators can be found in Refs [15, 16].

As it was mentioned earlier, one of the necessary conditions for the demonstration of stochastic resonance in a nonlinear system is the existence of metastable states, which the system can hop between with the aid of stochastic noise and a suitable modulation drive. Thus, in order to set a possible working point of the resonator at the metastable region, two preliminary hysteresis measurements were performed. In one measurement exhibited in Fig. 1 (a), forward and backward frequency sweeps of the reflection parameter $S_{11}$ were measured for the fundamental mode of the resonator at $f_{0} \simeq 2.57 \mathrm{GHz}$. The bidirectional frequency sweeps form two hysteresis

*Electronic address: baleegh@tx.technion.ac.il

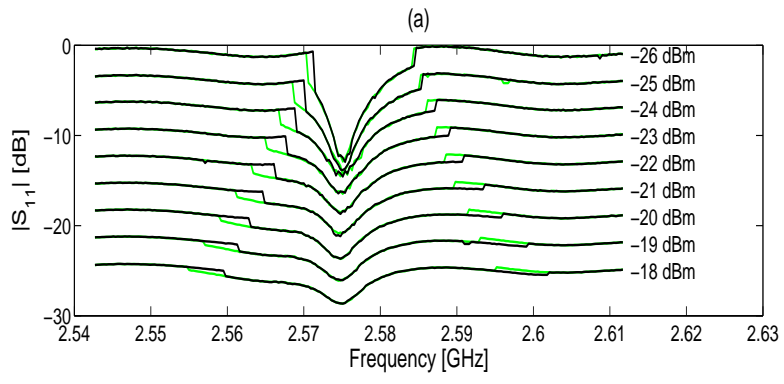

(b)

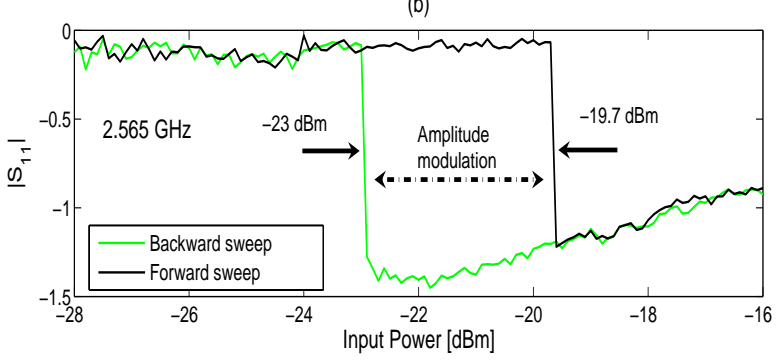

FIG. 1: (Color online). (a) Forward and backward frequency sweeps applied to the first mode of the resonator at $\sim 2.57 \mathrm{GHz}$. The sweeps exhibit hysteresis loops at both sides of the resonance line shape. The plots corresponding to different input powers were shifted by a vertical offset for clarity. (b) Reflected power hysteresis measured at a constant angular frequency of $\omega_{p}=2 \pi \cdot 2.565 \mathrm{GHz}$ which resides within the left-side metastable region of the resonance. For both plots the black (dark) line represents a forward sweep whereas the green (light) line represents a backward sweep.

loops at both sides of the resonance line shape at which the resonator becomes bistable. In another measurement shown in Fig. [1(b) the frequency of the pump $f_{p}$ was set to $2.565 \mathrm{GHz}$ positioned at the left side of the resonance, while the input power was swept in the forward and the backward directions. A hysteresis loop of the reflection parameter is apparent in this measurement as well, this time along the power axis. Thus, the working point was set to $f_{p}=2.565 \mathrm{GHz}, P_{0}=-21.5 \mathrm{dBm}$, while the applied modulation drive was a sinusoidal AM signal with a modulation amplitude $A_{\text {mod }}=0.27$.

A schematic diagram of the experimental setup em- 


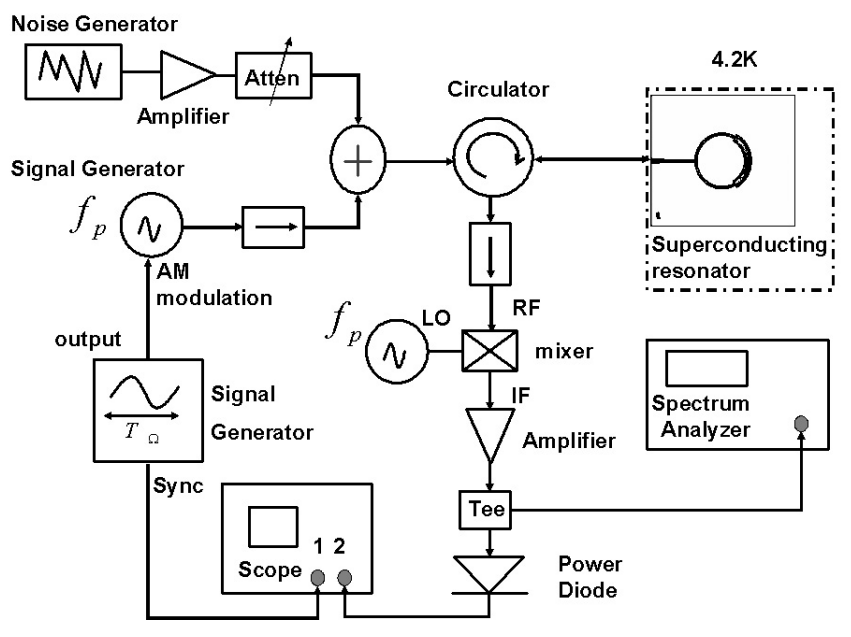

FIG. 2: Schematic drawing of the experimental setup used to measure stochastic resonance. The microwave signal generator and the local oscillator at frequency $f_{p}$ were phase locked. The layout of the resonator is shown at the top-right corner.

ployed to measure stochastic resonance is depicted in Fig. 2 A coherent signal $P_{0} \cos \left(\omega_{p} t\right)$ with angular frequency $\omega_{p}=2 \pi \cdot f_{p}$ is AM modulated using a sinusoidal generator with an angular frequency $\Omega$. The modulated signal is combined with white noise and injected into the resonator. The white noise which is generated using a noise source is amplified using an amplifying stage and tuned via an adjustable attenuator. The reflected power off the resonator is mixed with a local oscillator with frequency $f_{p}$ and measured simultaneously in the time and frequency domains via an oscilloscope and a spectrum analyzer respectively. Thus, the input signal power fed to the test port of the resonator (after calibrating the path losses) reads

$$
P_{\text {in }}(t)=P_{0}\left[1+A_{\mathrm{mod}} \sin (\Omega t+\varphi)\right] \cos \left(\omega_{p} t\right)+\xi(t),
$$

where $\xi(t)$ denotes a zero-mean Gaussian white noise $\langle\xi(t)\rangle=0$, with autocorrelation function $\left\langle\xi(t) \xi\left(t^{\prime}\right)\right\rangle=$ $2 D \delta\left(t-t^{\prime}\right)$, where $D$ is the noise intensity. For convenience, we choose the phase of the periodic drive to be zero $\varphi=0$.

For small amplitudes of the modulation signal $A_{\bmod } \ll$ 1 and in steady state conditions, the reflected power off the resonator $P_{r}(t)$ measured via a homodyne detection setup at the output, can in general, be written in the form [4, 5]

$$
P_{r}(t)=P_{0}^{r}+\sum_{n=1}^{\infty} A_{n}^{r}(D) \cos \left(n \Omega t+\phi_{n}(D)\right)+\xi^{r}(t),
$$

where $P_{r}(t)$ is a superposition of a dc component $P_{0}^{r}$, periodic functions of time with integer multiples of the fundamental angular frequency $\Omega$, and $\xi^{r}(t)$ the noise at the output. The coefficients $A_{n}^{r}(D)$ and $\phi_{n}(D)$ represent the reflected power amplitude and the phase lag of the $n$-th harmonic as a function of the noise intensity respectively. Calculating the phase-averaged spectral density of $P_{r}(t)$ yields

$$
\begin{aligned}
S(\omega) & =2 \pi P_{0}^{r} \delta(\omega) \\
& +\pi \sum_{n=1}^{\infty} A_{n}^{r}(D)[\delta(\omega-n \Omega)+\delta(\omega+n \Omega)]+S_{N}(\omega),
\end{aligned}
$$

which is composed of a delta spike at dc $(\omega=0)$, delta spikes with amplitudes $A_{n}^{r}(D)$ centered at $\omega= \pm n \Omega$, $n=1,2,3 \ldots$, and a background spectral density of the noise denoted by $S_{N}(\omega)$.

As it is known, one of the distinguished fingerprints of stochastic resonance phenomenon is a peak observed in the signal to noise ratio (SNR) curve as a function of the input noise intensity $D$, corresponding to some nonzero intensity $D_{\mathrm{SR}}$. This counterintuitive amplification in SNR curve is generally explained in terms of coherent interaction between the modulating signal and the stochastic noise entering the system.

In this framework a SNR for the $n$-th harmonic can be defined as [1]

$\mathrm{SNR}_{n}=2\left(\lim _{\Delta \omega \rightarrow 0} \int_{n \Omega-\Delta \omega}^{n \Omega+\Delta \omega} S(\omega) d \omega\right) / S_{N}(n \Omega)=\frac{2 \pi A_{n}^{r}(D)}{S_{N}(n \Omega)}$,

where SNR of the fundamental harmonic corresponds to $n=1$.

In Fig. 3 three SNR curves as a function of noise intensity are shown, corresponding to the fundamental $n=1$ and the odd harmonics $n=3$ and $n=5$. All three curves display a synchronized peak in the SNR around $D_{\mathrm{SR}}$ of about $2 \mathrm{fW} / \mathrm{Hz}$.

Typical results of stochastic resonance measured in the time and the frequency domains are shown at the left and right sides of Fig. 固respectively. Panels (a) and (b) correspond to low noise levels below the critical value. Panel (a) shows the reflected sinusoidal at $f_{\Omega}=1 \mathrm{kHz}$ without jumps. Panel (b) shows the reflected sinusoidal containing a few arbitrary jumps. Whereas, panel (c) which corresponds to the critical stochastic noise $D_{\mathrm{SR}}$ exhibits one jump in the reflected signal at every half cycle. That is, the time-scale matching condition for stochastic resonance given by $\tau\left(D_{\mathrm{SR}}\right)=T_{\Omega} / 2$, is fulfilled for this noise intensity, where $T_{\Omega}=2 \pi / \Omega$, and $\tau(D)$ is the metastable state lifetime corresponding to the noise intensity $D$. A possible theoretical linkage between the transition rate $1 / \tau$ exhibited by these resonators and their physical nonlinear mechanism will be established in a future publication [17]. In panels (d) and (e) on the other hand, the case of noise levels higher than $D_{\mathrm{SR}}$ are shown. In panel (d), the coherence between the modulating drive and the noise is lost, and multiple jumps are observed during each 


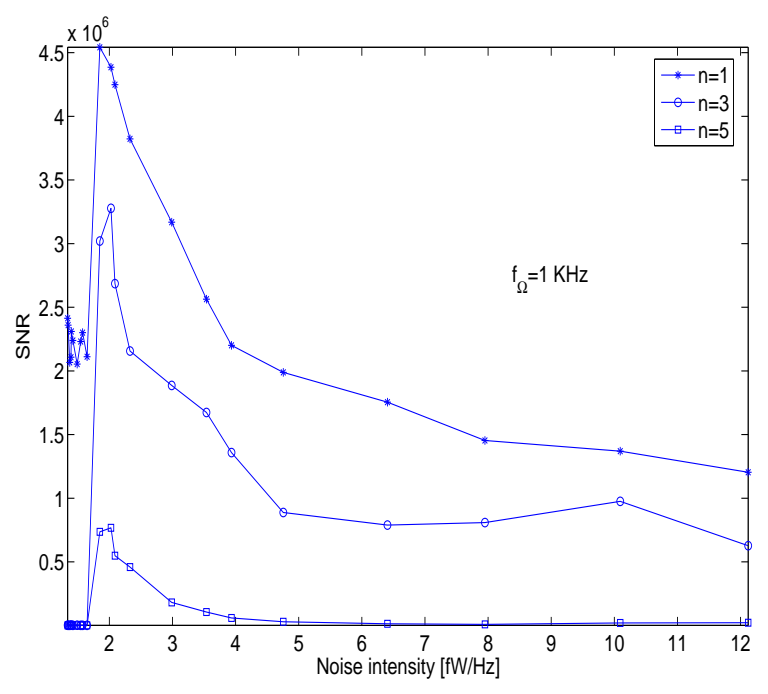

FIG. 3: SNR curves measured for the fundamental $n=1$ and the odd harmonics $n=3, n=5$, as a function of the input noise intensity. A peak in the SNR is detected at around $D_{\mathrm{SR}}$ of $2 \mathrm{fW} / \mathrm{Hz}$.
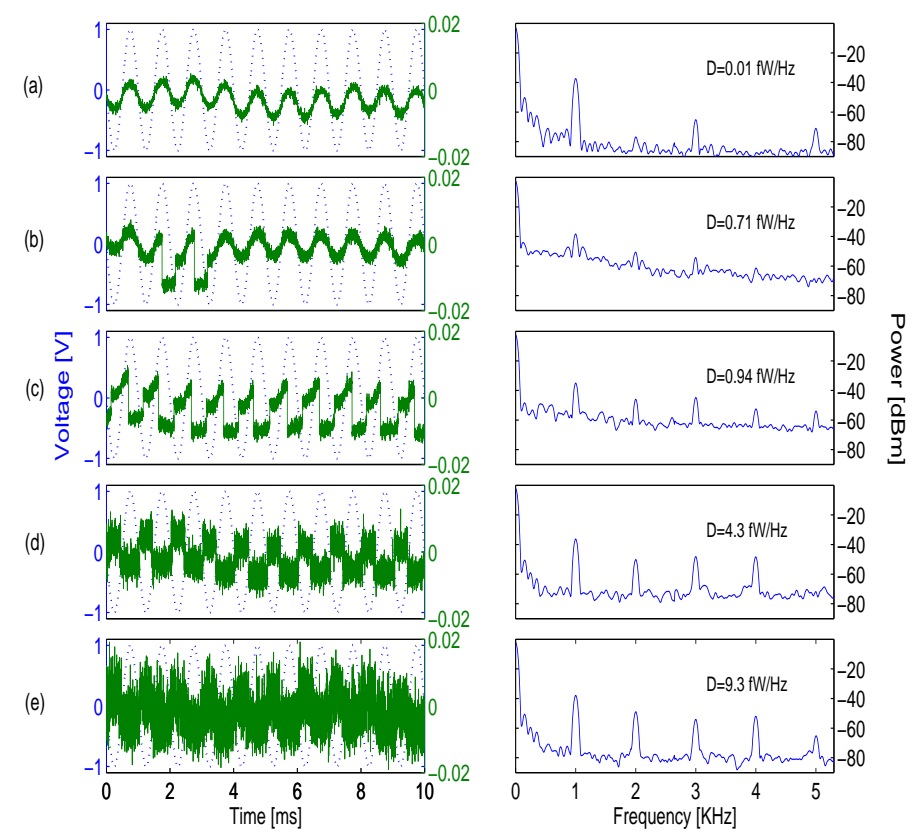

FIG. 4: (Color online). Panels (a)-(e) exhibit typical snapshots of the reflected modulated signal in the time domain (left side) and in the frequency domain (right side) as the input noise intensity $D$ is increased. Panels (a)-(b) correspond to noise intensities below $D_{\mathrm{SR}}$. Panel (c) corresponds to a noise intensity of $D_{\mathrm{SR}}=0.94 \mathrm{fW} / \mathrm{Hz}$. Panels (d)-(e) correspond to noise intensities higher than $D_{\mathrm{SR}}$. The dotted sinusoidal line drawn in the time domain represents the modulation signal. cycle. Finally, in panel (e), the high noise almost screens the signal and the induced jumps are totally uncorrelated. It is also apparent that the reflected modulated signals shown in panels (c) and (d), in addition to being amplified, exhibit a rather rectangular shape. Such distortion to the sinusoidal shape at the output may originate from a dispersive character of the system response as was suggested in Refs. [5, 18].

In the frequency domain displayed at the right side of panels (a)-(e), the fundamental and the harmonics up to $n=5$ are shown, as the noise intensity is increased. The appearance of even harmonics in the frequency spectrum indicates that the nonlinear system is asymmetric, in contrast to symmetric potential systems where only the odd harmonics emerge [4, 19].

Furthermore, if one defines a spectral amplification of the $n$-th harmonic spectral density relative to that of the fundamental measured for the noiseless case, one gets

$$
\eta_{n}(D)=A_{n}^{r}(D) / A_{1}^{r}(D=0),
$$

where the amplitude $A_{1}^{r}(D=0)$ is assumed to be proportional to the input power of the modulation signal up to some constant reflection factor. Since the different harmonics of the signal are strongly correlated, we choose in the following figures to exhibit the amplification of the third harmonic instead of the fundamental one $n=1$. This is mainly because the first harmonic resides at the frequency of the modulation source signal and part of it bypasses the resonator.

In Fig. 5 the amplification parameter $\eta_{3}$ of the third harmonic as a function of the input noise intensity was

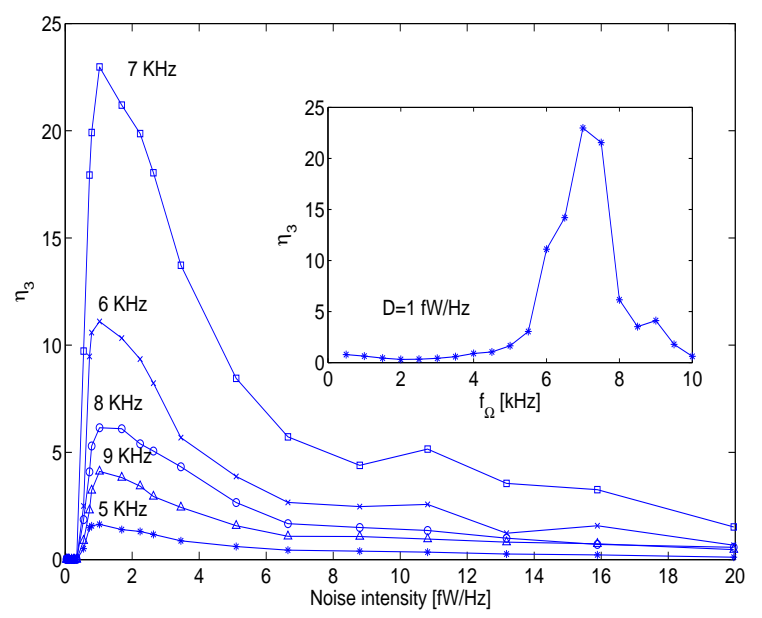

FIG. 5: Third harmonic amplification $\eta_{3}$ as defined in Eq. 5 plotted as a function of input noise intensity. The different plots correspond to $f_{\Omega}=5,6,7,8,9 \mathrm{kHz}$ modulation frequencies. The inset shows $\eta_{3}$ gain as a function of $f_{\Omega}$, corresponding to a constant input noise intensity of $1 \mathrm{fW} / \mathrm{Hz}$. 


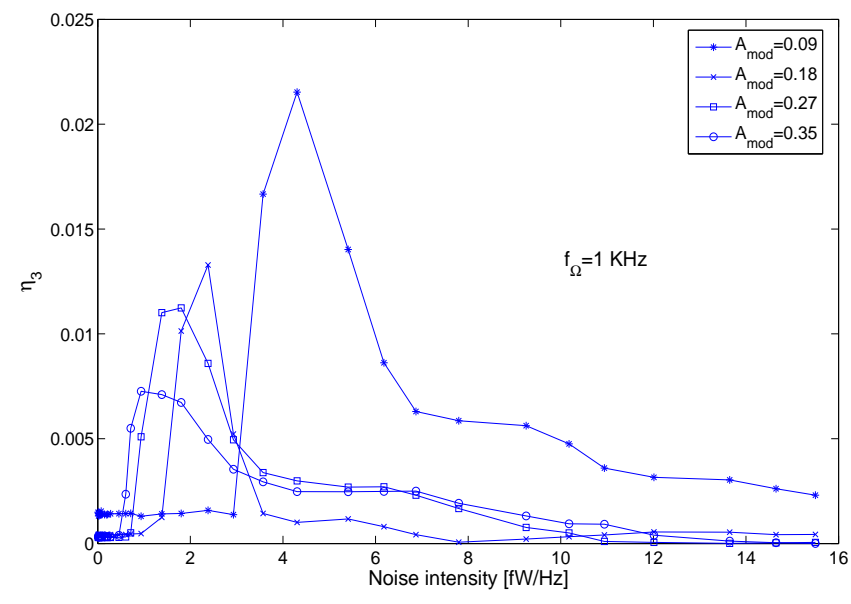

FIG. 6: Third harmonic amplification $\eta_{3}$ as defined in Eq. 5 plotted as a function of input noise intensity at $f_{\Omega}=1 \mathrm{kHz}$. The different plots correspond to an increasing modulation amplitude $A_{\text {mod }}=0.09,0.18,0.27,0.35$.

measured at a fixed modulation amplitude but for different AM drive frequencies $f_{\Omega}=5,6,7,8,9 \mathrm{kHz}$. As it is apparent from the figure $\eta_{3}$, amplification is nonmonotonic upon increasing the forcing frequency $f_{\Omega}$. The amplification graphs rise up with frequency up to $7 \mathrm{kHz}$ and afterwards decay down. A cross section of $\eta_{3}$ parameter at a constant noise intensity of $1 \mathrm{fW} / \mathrm{Hz}$ is shown in the inset as a function of $f_{\Omega}$ varying in the range $0.5-10 \mathrm{kHz}$. A peak in $\eta_{3}$ is observed at $f_{\Omega}=7.5 \mathrm{kHz}$. Similar amplification behavior was observed for the fundamental and for the fifth harmonic as well. However, this non-monotonic behavior of $\eta_{3}$ as a function of the forcing frequency somehow departs from the monotonic decrease presented for example in Refs. 1, 4]. This may be due to the nature of the nonlinearity governing our system [16] which can not be explained in terms of Duffing oscillator nonlinearity, that was assumed in the cited papers. A theoretical prediction of a non-monotonic SNR versus $f_{\Omega}$ was introduced though in Ref. [20], where the case of two square potential wells separated by a square barrier was considered.

In contrast, when the modulation amplitude is increased at a fixed forcing frequency, the resultant amplification is expected to decrease, mainly due to increased nonlinear effects at higher modulation amplitudes, which tend to suppress stochastic resonance phenomenon [4]. A good confirmation to this hypothesis is presented in Fig. [6] which displays decaying amplification curves, as a function of the noise intensity, upon increasing the modulation amplitudes $A_{\text {mod }}=0.09,0.18,0.27,0.35$ at a fixed forcing frequency of $f_{\Omega}=1 \mathrm{kHz}$.

In conclusion, nonlinear $\mathrm{NbN}$ superconducting resonators have been shown to exhibit stochastic resonance when driven into the bistable region. Amplification of a slowly varying AM signal carried by a microwave pump can be achieved by establishing a resonant cooperation between the modulating signal and the injected stochastic noise. Such amplification scheme may be useful in the detection and amplification of small AM signals in communication area. Moreover, stochastic resonance phenomenon can be employed to some extent, to "probe" some important characteristics of the nonlinearity mechanism of the resonators [17].

This work was supported by the German Israel Foundation under grant 1-2038.1114.07, the Israel Science Foundation under grant 1380021, the Deborah Foundation, the Poznanski Foundation, and MAFAT.
[1] L. Gammaitoni, P. Hänggi, P. Jung, and F. Marchesoni, Rev. Mod. Phys. 70, 223 (1998).

[2] A. R. Bulsara and L. Gammaitoni, Physics Today p. 39 (March 1996).

[3] L. Gammaitoni, F. Marchesoni, and S. Santucci, Phys. Rev. Lett. 74, 1052 (1995).

[4] P. Jung, and P. Hänggi, Phys. Rev. A 44, 8032 (1991).

[5] M. Morillo and J. Gómez-Ordóñez, Phys. Rev. E 51, 999 (1995).

[6] R. Benzi, A. Satera, G. Parisi, and A. Vulpiani, J. Phys. A 14, L453 (1981).

[7] R. Benzi, A. Satera, G. Parisi, and A. Vulpiani, SIAM J. Appl. Math. 43, 565 (1983).

[8] S. Fauve and F. Heslot, Phys. Lett. A 97, 5 (1983).

[9] A. Longtin, A. Bulsara, and F. Moss, Phys. Rev. Lett. 67, 656 (1991).

[10] J. E. Levin and J. P. Miller, Nature 380, 165 (1996).

[11] B. McNamara, K. Wiesenfeld, and R. Roy, Phys. Rev. Lett. 60, 2626 (1988).

[12] R. Rouse, S. Han, and J. E. Lukens, Appl. Phys. Lett.
66, 108 (1995).

[13] A. D. Hibbs, A. L. Singsaas, E. W. Jacobs, A. R. Bulsara, J. J. Pekkedahl, and F. Moss, J. Appl. Phys. 77, 2582 (1995).

[14] R. L. Badzey and P. Mohanty, Nature 437, 995 (2005).

[15] B. Abdo, E. Arbel-Segev, O. Shtempluck, and E. Buks, cond-mat/0501114 to be published in IEEE Trans. Appl. Supercond.

[16] B. Abdo, E. Arbel-Segev, O. Shtempluck, and E. Buks, Phys. Rev. B 73, 134513 (2006).

[17] B. Abdo, E. Arbel-Segev, O. Shtempluck, and E. Buks, manuscript in preparation.

[18] M. I. Dykman, D. G. Luchinsky, R. Mannella, P. V. E. McClintock, N. D. Stein, and N. G. Stocks, J. Stat. Phys. 70, 463 (1993).

[19] M. E. Inchiosa, A. R. Bulsara, and L. Gammaitoni, Phys. Rev. E 55, 4049 (1997).

[20] V. Berdichevsky and M. Gitterman, J. Phys. A 29, L447 (1996). 